\title{
Pneumonectomy for lung cancer in the elderly: lessons learned from a multicenter study
}

\author{
Fabrizio Minervini ${ }^{1 \#} \wedge$, Gregor J. Kocher ${ }^{2 \#} \wedge$, Pietro Bertoglio ${ }^{3 \wedge}$, Peter B. Kestenholz ${ }^{1 \wedge}$, \\ Carlos Gálvez Muñoz ${ }^{4}$, Davide Patrini ${ }^{5} \wedge$, Laurens J. Ceulemans ${ }^{6,7}$, Housne Begum ${ }^{8}$, Jon Lutz ${ }^{2}$, \\ Max Shojai ${ }^{2}$, Yaron Shargall ${ }^{8} \wedge$, Marco Scarci ${ }^{9} \wedge$
}

${ }^{1}$ Department of Thoracic Surgery, Cantonal Hospital Lucerne, Lucerne, Switzerland; ${ }^{2}$ Division of Thoracic Surgery, Bern University Hospital, University of Bern, Bern, Switzerland; ${ }^{3}$ Division of Thoracic Surgery, IRCSS Azienda Ospedaliero-Universitaria, Bologna, Italy; ${ }^{4}$ Department of Thoracic Surgery, University Hospital Alicante, Alicante, Spain; ${ }^{5}$ Department of Thoracic Surgery, University College London Hospitals, London, UK; ${ }^{6}$ Department of Thoracic Surgery, University Hospitals Leuven, Leuven, Belgium; ${ }^{7}$ Department of Chronic Diseases and Metabolism, Laboratory BREATHE, KULeuven, Leuven, Belgium; ${ }^{8}$ Department of Thoracic Surgery, McMaster University, Hamilton, Canada; ${ }^{9}$ Department of Thoracic surgery, San Gerardo Hospital, Monza, Italy

Contributions: (I) Conception and design: F Minervini, M Scarci; (II) Administrative support: F Minervini; (III) Provision of study materials or patients: All authors; (IV) Collection and assembly of data: F Minervini, P Bertoglio; (V) Data analysis and interpretation: F Minervini, P Bertoglio; (VI) Manuscript writing: All authors; (VII) Final approval of manuscript: All authors.

\#These authors contributed equally to this work.

Correspondence to: Fabrizio Minervini, MD, PhD. Department of Thoracic Surgery, Cantonal Hospital Lucerne, Lucerne, Switzerland. Email: fabriziominervini@hotmail.com.

Background: $60 \%$ of patients diagnosed with lung cancer are older than 65 years and are at risk for substandard treatment due to a reluctance to recommend surgery. Pneumonectomy remains a high risk procedure especially in elderly patients. Nevertheless, the impact of age and neoadjuvant treatment on outcomes after pneumonectomy is still not well described.

Methods: We performed a multicentric retrospective study, analyzing outcomes of patients older than 70 years who underwent pneumonectomy for central primary lung malignancy between January 2009 and June 2019 in 7 thoracic surgery departments: Lucerne and Bern (Switzerland), Hamilton (Canada), Alicante (Spain), Monza (Italy), London (UK), Leuven (Belgium). Survival was estimated with Kaplan-Meier, and differences in survival were determined by log-rank analysis. We investigated pre- and post-operative prognostic factors using Cox proportional hazards regression model; multivariable analysis was performed only with variables, which were statistically significant at the invariable analysis.

Results: A total of 136 patients were included in the study. Mean age was 73.8 years (SD 3.6). 24 patients $(17.6 \%)$ had an induction treatment (chemotherapy alone in 15 patients and chemo-radiation in 9). Mean length of stay (LOS) was 12.6 days (SD 10.39) and 74 patients $(54.4 \%$ ) had experienced a post-operative complication: 29 (21.3\%) had a pulmonary complication, 33 (24.3\%) had a cardiac complication and in 12 cases $(8.8 \%)$ patients experienced both cardiac and pulmonary complications. 16 patients were readmitted [median LOS 13.7 days (range, 2-39 days)] and of those 14 (10.3\%) required redo surgery. Median overall survival (OS) of the entire cohort was 38 months (95\% CI: 29.9-46.1 months); in-hospital mortality was $1.5 \%$, 30-day mortality rate was $3.7 \%$, while 90 -day mortality was $8.8 \%$ accounting for 5 and 12 patients respectively. Patients receiving neo-adjuvant therapy did not experience a higher incidence of postoperative complications $(\mathrm{P}=0.633)$, did not have a longer postoperative course $(\mathrm{P}=0.588)$, nor did they have an

^ ORCID: Fabrizio Minervini, 0000-0003-3595-7307; Gregor J. Kocher, 0000-0003-1586-6774; Pietro Bertoglio, 0000-0002-6267-0099; Peter B. Kestenholz, 0000-0002-0419-3685; Carlos Gálvez Muñoz, 0000-0002-1226-0841; Davide Patrini, 0000-0002-2492-799X; Laurens J. Ceulemans, 0000-0002-4261-7100; Jon Lutz, 0000-0001-6622-6924; Yaron Shargall, 0000-0001-8585-0327; Marco Scarci, 0000-00024459-7721. 
increased mortality rate $(\mathrm{P}=0.863)$.

Conclusions: Age should not be considered an absolute contraindication for pneumonectomy in elderly patients even after neoadjuvant treatment. It has become apparent that especially in these patients, a patienttailored approach with a careful selection should be used to define the risk-benefit balance.

Keywords: Pneumonectomy; lung surgery in the elderly; lung cancer in the elderly

Submitted May 24, 2021. Accepted for publication Sep 08, 2021.

doi: $10.21037 /$ jtd-21-869

View this article at: https://dx.doi.org/10.21037/jtd-21-869

\section{Introduction}

Current clinical guidelines for the treatment of non-small cell lung cancer (NSCLC) recommend anatomic pulmonary resection with lobectomy as the gold standard (1). During the last decades, despite an increased proficiency in sleeve resections, pneumonectomy remains, at times, the only approach to achieve an R0 resection in extended primary lung cancers. When pneumonectomy is the only surgical option, different factors such as disease stage, lung function, co-morbidities and age have to be taken into considerations in order to propose the best treatment, especially in elderly patients.

About $60 \%$ of patients diagnosed with pulmonary cancer are older than 65 years and most cancer-related deaths occur within this age population (2).

Commonly, the most used cutoff to define elderly patients is an age of 70 and above (3). There is evidence that older patients are at risk for substandard treatment when compared to the younger counterparts due to a reluctance to choose surgical approach because of the concerns regarding pre-existing comorbidities, outcomes and limited life expectancy (4).

However, as the median life expectancy of populations among industrialized countries has significantly increased in recent years providing optimal treatment for elderly patients has become a key challenge. Even though video assisted thoracoscopic surgery (VATS) techniques along with Enhanced Recovery After Surgery (ERAS) programs allowed to reduce the risk of complications' pneumonectomy presumably remains a high risk procedure especially in elderly patients (5). Nevertheless, the impact of age on pneumonectomy's outcomes has not been well analyzed.

We present the following article in accordance with the STROBE reporting checklist (available at https://dx.doi. org/10.21037/jtd-21-869).

\section{Methods}

\section{Data source}

In this international retrospective cohort study design, data from January 2009 to December 2019 was collected from seven thoracic surgery centers at Lucerne Cantonal Hospital (Lucerne, Switzerland), St. Joseph's Healthcare Hamilton, McMaster University (Hamilton, Canada), University College London (London, UK), University Hospital Alicante (Alicante, Spain), University Hospital Bern (Bern, Switzerland), University Hospital Leuven (Leuven, Belgium) and San Gerardo Hospital (Monza, Italy). Each center accessed their local, prospectivelycollected institutional databases to obtain the data, along with an additional retrospective chart review of patient medical records in order to complete missing data. The study was conducted in accordance with the Declaration of Helsinki (as revised in 2013). The study was approved by the Ethics Board Central Switzerland, project \#201902258 and University Hospitals Leuven, Belgium project S63068. Individual consent for this retrospective analysis was waived. All patients older than 70 years who underwent an elective pneumonectomy via either thoracotomy or minimally invasive approaches [video-assisted thoracoscopic surgery (VATS) or robotic resections] were identified in the databases and screened for inclusion criteria. Patients were included if they were older than 70 years and their resections were for primary or metastatic lung malignancies. Patients were excluded if they underwent a pneumonectomy in an emergency setting or for a benign disease.

\section{Outcomes}

The primary outcomes were in-hospital morbidity and inhospital mortality. Secondary outcomes included: hospital readmission, need for re-intervention, 30-day mortality, 
Table 1 Patient characteristics

\begin{tabular}{lc}
\hline Characteristic & Outcome \\
\hline Gender (male), n (\%) & $97(71.3)$ \\
Mean age ( \pm SD) & $73.9( \pm 3.6)$ \\
Mean FEV1 (L, \pm SD) & $2.2( \pm 0.6)$ \\
Mean FEV1\% ( \pm SD) & $79.2( \pm 18.9)$ \\
Mean DLCO\% ( \pm SD) & $70.8( \pm 18.6)$ \\
Cardiac morbidity (yes), n (\%) & $69(50.7)$ \\
Cardiac comorbidity details, n (\%) & \\
Arrhythmia & $9(6.5)$ \\
CAD & $12(8.8)$ \\
Hypertension & $41(30.2)$ \\
Previous cardiac surgery & $1(0.7)$ \\
Surgical approach, n (\%) & \\
Thoracotomy & $125(91.9)$ \\
Sternotomy & $2(1.5)$ \\
VATS & $9(6.6)$ \\
Side (right), $n(\%)$ & $48(35.3)$ \\
Neoadjuvant (yes), n (\%) & $24.6)$ \\
\hline
\end{tabular}

FEV1, forced expiratory volume in the 1st second; DLCO, diffusing capacity for carbon monoxide; CAD, coronary artery disease; VATS, video assisted thoracoscopic surgery.

90-day mortality, and 1-, 3-, 5-year survival rates, respectively. Data was collected on patients age, sex, forced expiratory volume in 1 second (FEV1), diffusion lung capacity for carbon monoxide) (DLCO) measurements from pre-operative pulmonary function testing, total length of stay (LOS) and postoperative complications.

\section{Statistical analysis}

Continuous variables are reported as mean and standard deviation in normally distributed data; discrete variables are reported as numbers and percentage. A $\mathrm{P}$ value of $<0.05$ was considered to be of statistical significance. Survival was estimated with Kaplan-Meier, and differences in survival were determined by log-rank analysis. Pre- and post-operative prognostic factors were investigated using Cox proportional hazards regression model; multivariable analysis was performed only with variables, which were statistically significant at the univariable analysis. The hazard ratio (HR) and 95\% confidence intervals (CI) were reported for covariates.

All data were de-identified with a sequentially-generated study identification code, encrypted, and transferred to the central Lucerne site for analysis. STATA v14 (StataCorp, Texas, USA, www.stata.com) and SPSS 25.0 (www.ibm.com) were used for statistical analysis.

\section{Results}

A total of 136 patients were included in the study. Patients' characteristics are summarized in Table 1. Mean age was 73.8 (SD 3.6), median age was 73. Preoperatively pulmonary and cardiovascular assessment (including transthoracic echocardiography) were performed in all patients.

A total of 112 patients (82.4\%) did not undergo neoadjuvant therapy, while the remaining 24 patients had an induction treatment (chemotherapy alone in 15 patients and chemo-radiotherapy in 9). Overall, pulmonary resection was performed via a thoracotomy in 127 patients $(93.4 \%)$ and through a minimally invasive (VATS) approach in 9 patients (6.6\%). 48 patients (35.3\%) underwent right sided resection whereas $88(64.7 \%)$ underwent left sided one.

Mean LOS was 12.6 days $(S D \pm 10.39)$ with a median of 9 days (range, 3-65 days) and 74 patients (54.4\%) experienced post-operative complications: $29(21.3 \%)$ had a pulmonary complication (4 grade I, 20 grade II and 5 grade III according to the Common Terminology Criteria for Adverse Events), 33 (24.3\%) had cardiac complications (10 grade I, 20 grade II, 2 grade III and 1 grade IV) and in 12 cases (8.8\%) patients experienced both cardiac and pulmonary complications (65\% grade II complications). 16 patients were readmitted [median LOS 13.7 days (range, 2-39 days)] and of those 14 $(10.3 \%)$ required redo surgery (2 due to hemothorax and 12 because of bronchopleural fistula with post-pneumonectomy empyema). Two patients died in hospital, resulting in an inhospital mortality rate of $1.5 \%$. 30-day mortality rate was $3.7 \%$, while 90 -day mortality was $8.8 \%$ accounting for 5 and 12 patients respectively.

In the examined period, considering all the centers, 418 pneumonectomies were performed with an in-hospital mortality rate of $1.2 \%$ and 30 -day mortality rate of $2.9 \%$.

Postoperative outcomes, including complications, were in our analysis not related to neo-adjuvant therapy; more in details, patients receiving neo-adjuvant therapy did not experience a higher incidence of postoperative complications $(\mathrm{P}=0.633)$, did not have a longer postoperative hospital stay $(\mathrm{P}=0.588)$, nor did they have a higher in-hospital mortality 
Table 2 Patient's outcomes

\begin{tabular}{|c|c|}
\hline Variable & Outcome \\
\hline Mean length of stay, day $( \pm$ SD) & $12.6(10.4)$ \\
\hline \multicolumn{2}{|l|}{ In-hospital complications, n (\%) } \\
\hline Yes & $74(54.4)$ \\
\hline Pulmonary & $29(21.3)$ \\
\hline Cardiac & $33(24.3)$ \\
\hline More than one & $12(8.8)$ \\
\hline \multicolumn{2}{|l|}{ Pulmonary complications, $\mathrm{n}(\%)$} \\
\hline Acute respiratory failure & $4(2.4)$ \\
\hline ARDS & $1(0.7)$ \\
\hline Broncho-pleural fistula & $2(1.4)$ \\
\hline Pneumonia & $20(14.7)$ \\
\hline Empyema & $8(5.9)$ \\
\hline Pulmonary embolism & $3(2.1)$ \\
\hline Other & $1(0.7)$ \\
\hline \multicolumn{2}{|l|}{ Cardiac complications, n (\%) } \\
\hline Atrial fibrillation & $30(22.2)$ \\
\hline Myocardial infarction & $3(2.2)$ \\
\hline Cardiac failure & $4(2.9)$ \\
\hline Other & $8(5.8)$ \\
\hline Redo surgery (yes), n (\%) & $14(10.3)$ \\
\hline Readmission (yes) & $16(11.8)$ \\
\hline $\begin{array}{l}\text { Mean length of stay during readmission, } \\
\text { days }( \pm S D)\end{array}$ & $13.7( \pm 10.2)$ \\
\hline \multicolumn{2}{|l|}{ Pathological stage (TNM 7th edition), n (\%) } \\
\hline IA & $13(9.6)$ \\
\hline IB & $15(11.0)$ \\
\hline IIA & $6(4.4)$ \\
\hline IIB & $40(29.4)$ \\
\hline IIIA & $46(33.8)$ \\
\hline IIIB & $10(7.4)$ \\
\hline IV & $4(2.9)$ \\
\hline \multicolumn{2}{|l|}{ Adjuvant treatment, $\mathrm{n}(\%)$} \\
\hline Yes & $38(28)$ \\
\hline Chemotherapy & $17(12.5)$ \\
\hline Radiotherapy & $2(1.5)$ \\
\hline Chemoradiotherapy & $10(7.4)$ \\
\hline Other & $9(6.6)$ \\
\hline
\end{tabular}

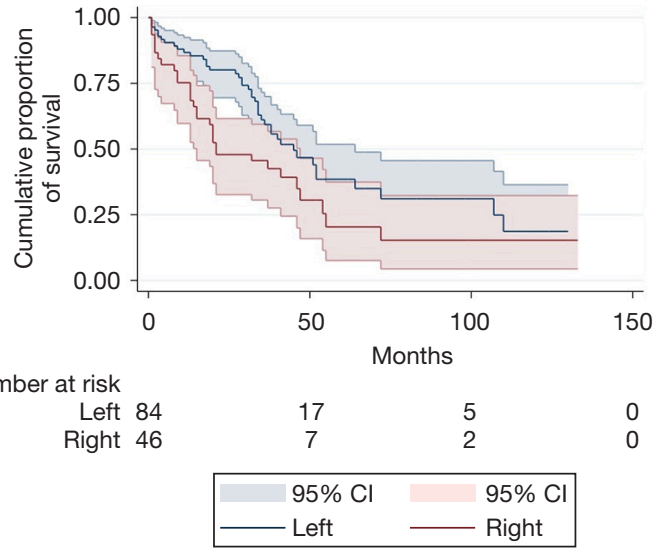

Figure 1 Influence of operated side on OS. OS, overall survival.

rate $(\mathrm{P}=0.863)$ (Table 2).

Fourteen patients $(58.3 \%)$ who received induction therapy had a downstaging afterward.

With a median follow up of 83 months, median overall survival (OS) of the entire cohort was 38 months (95\% CI: 29.9-46.1 months); stage specific survival was 52 months (95\% CI: 14.1-89.9 months) for stage I, 35 months (95\% CI: 27.0-43.0 months) for stage II, 38 months (95\% CI: 23.3-52.7 months) for stage III and 8 months (95\% CI: non calculable) for stage IV. Survival rates at 1-, 3- and 5-years were $77 \%, 52 \%$ and $31 \%$ respectively.

As expected, we observed a better prognosis after left pneumonectomy (Figure 1).

Univariable analysis of prognostic factors influencing OS revealed that preoperative cardiac morbidity $(\mathrm{P}=0.048)$, postoperative complications $(\mathrm{P}=0.012)$ and more specifically pulmonary complications ( $\mathrm{P}=0.015$, Figure 2$)$ were significant prognostic factors for reduced OS (Table 3). Interestingly an increased LOS (also in case of readmission) was significantly correlated with a worse OS. Nevertheless, none of the above-mentioned prognostic factors significantly influenced OS in a multivariable analysis.

\section{Discussion}

Considering that the global population is constantly getting older and older, the surgical demand in the elderly is gaining importance. Even if in 2003 lung cancer was reported as second leading cause of cancer death in octogenarians, often geriatric patients are not a priori considered suitable for surgery because of concerns regarding age-related comorbidities and outcomes (6). 


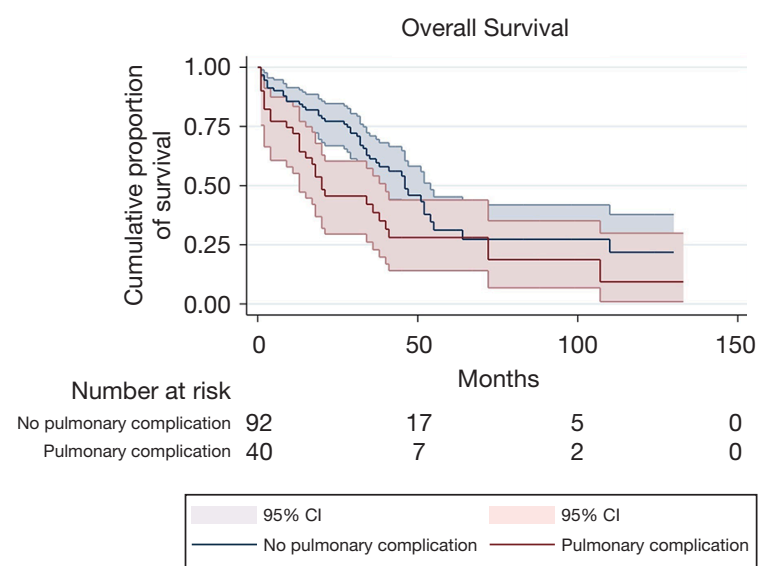

Figure 2 Influence of pulmonary complications on OS. OS, overall survival.

In the literature, the operative mortality related to any lung resection in the elderly ranges from $0 \%$ to $8 \%$ (7). Many, but not all studies reported a correlation between pneumonectomy and peri-operative mortality rate. Bernet et al. reported a mortality of $3.8 \%$ in the pneumonectomy group, although it is noted that the number of analyzed patients was small $(\mathrm{n}=25)(8)$.

Yamamoto et al. found no significant difference in overall recurrence rate or long-term survival in elderly patients compared to younger ones. The authors attributed these results to a careful pre-operative patient selection (9).

Sirbu et al. performed a retrospective analysis of lung resection for NSCLC in geriatric patients. Among lung resections, 42 pneumonectomies (15 on the right side and 27 on the left side) were included reporting a $7.1 \%$ mortality and $24.5 \% 5$-year survival. However, they tried to avoid pneumonectomy in their cohort of patients whenever possible in order to maintain in-hospital mortality at an acceptable level (10).

Kiser and Detterbeck analyzed data from several studies for elderly patients who underwent surgery for lung cancer and found a mortality rate after pneumonectomy ranging from $16 \%$ to $25 \%$ (11). Myrdal et al. reported a major complication rate of $18.5 \%$ after pneumonectomy in patients older than 70 years undergoing a pneumonectomy with a 30 day-mortality of $5.7 \%$. Schneider et al. found a decreased postdischarge survival rate over the 90 days with the highest proportional mortality in the 60- to 90-day window $(32.3 \%$ mortality observed in the $71-75$ age group, $22.6 \%$ in the $76-80$ age group (12).

While the reported data showed controversial results,
Table 3 Prognostic factors influencing OS

\begin{tabular}{lcc}
\hline Prognostic factors & \multicolumn{2}{l}{ Univariable } \\
\cline { 2 - 3 } & $\mathrm{P}$ & $\mathrm{HR}(95 \% \mathrm{Cl})$ \\
\hline Male gender & 0.469 & $1.206(0.727-1.999)$ \\
Left side & $0.045^{\star}$ & $0.626(0.396-0.990)$ \\
Age & 0.786 & $1.009(0.945-1.078)$ \\
FEV1\% & 0.841 & $1.001(0.988-1.015)$ \\
DLCO\% & 0.082 & $0.987(0.973-1.002)$ \\
Cardiac morbidity & $0.048^{\star}$ & $1.596(1.004-2.539)$ \\
Open vs. VATS & 0.612 & $0.805(0.347-1.863)$ \\
Neoadjuvant & 0.402 & $1.283(0.716-2.298)$ \\
Lenght of stay & $0.007^{*}$ & $1.023(1.006-1.040)$ \\
Inhospital comorbidities & $0.012^{*}$ & $1.824(1.144-2.908)$ \\
Inhospital pulmonary & $0.015^{*}$ & $1.773(1.116-2.815)$ \\
comorbidities & & \\
Inhospital cardiac morbidity & 0.198 & $1.367(0.849-2.199)$ \\
Redo surgery & 0.662 & $1.160(0.596-2.256)$ \\
Pathological stage & 0.255 & $1.171(0.892-1.537)$ \\
Readmission & 0.717 & $1.149(0.542-2.437)$ \\
Length of stay of readmission & $0.018^{*}$ & $1.119(1.019-1.228)$ \\
Adjuvant & 0.859 & $0.942(0.487-1.822)$ \\
\hline$*$ P<0.05. OS, overals & & \\
\hline & &
\end{tabular}

*, $\mathrm{P}<0.05$. OS, overall survival; FEV1, forced expiratory volume in the 1st second; DLCO, diffusing capacity for carbon monoxide; VATS, video assisted thoracoscopic surgery.

it should be noted that the included studies were mostly retrospective analyses with small numbers of patients and sometimes very outdated. Surgery per se carries a risk of complications but what should be taken into account is also the influence of peri- and post-operative management on morbidity and mortality rates. Originally described for colorectal surgery, ERAS programs have been shown to substantially improve outcomes in the field of thoracic surgery as well (13-16). In our analysis, ERAS principles were part of the standard post-operative management across all sites. For this reason, in our opinion, an evaluation of a surgical treatment benefit should be based on and include an optimal and updated state-of-the-art post surgery enhanced recovery program. However, it must be noticed that the influence of ERAS on pneumonectomy patients has not been extensive evaluated. 


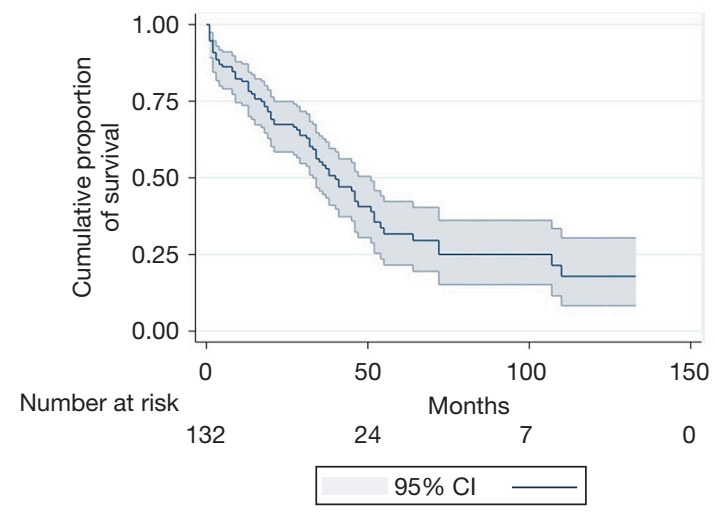

Figure 3 Whole population survival.

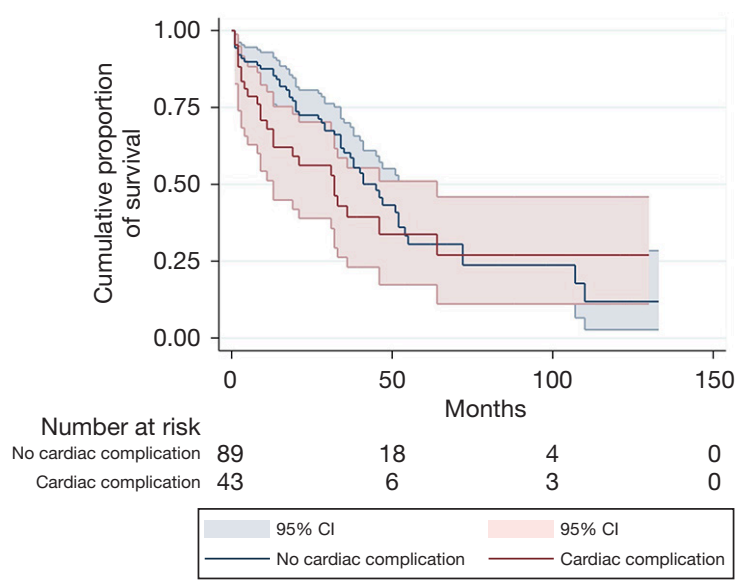

Figure 4 Influence of cardiac complication on OS. OS, overall survival.

Our analysis is quite recent and 136 patients were included, with $1.5 \%$ in-hospital mortality, $3.7 \% 30$ day mortality and $8.8 \% 90$-day mortality, which is lower when compared to the reported literature. The increase of mortality after discharge in our patients is consistent with other reports even if the causes of the problem have been so far overlooked and still remain unclear $(12,17,18)$.

The identification of prognostic factors for in hospital mortality and post-discharge mortality should be in depth evaluated in order to guide peri and postoperative management.

We observed high survival rates in our study contrary to what has been so far reported in the literature (Figure 3). Our results are probably influenced by the high proportion (54.4\%) of stage I/II patients (where a pneumonectomy was necessary due to the location of the tumor) along with a careful selection of the patients who underwent surgery. Even if previous studies showed that prognosis is influenced by $\mathrm{N}$ status and pathological stage, in our analysis, maybe due to the heterogeneous distribution with a minority of advanced stages or $\mathrm{N} 2$ status, we did not find a correlation between pN0-1 and pN2 or stage I-II versus III-IV (19). Sakakura et al. showed that completeness of resection $(\mathrm{P}=0.003)$ and subcategorization of involved neighboring organs $(\mathrm{P}=0.008)$ were more crucial predictors than the presence or absence of mediastinal lymph node metastasis $(\mathrm{P}=0.033)(20)$.

Pre-operative cardiac morbidity along with postoperative complication rates (particularly pulmonary complications) have significantly reduced OS in our analysis. No correlation was observed between cardiac complications and OS $(\mathrm{P}=0.192)$ (Figure 4).

Despite several studies identified induction therapy as negative prognostic factors on morbidity and mortality, in our analysis, patients receiving neoadjuvant treatment had similar outcomes to those who underwent pneumonectomy without induction (21-23). Similar encouraging results were published by Weder et al. who stated that pneumonectomy after neoadjuvant chemotherapy or chemoradiotherapy can be completed safely with a mortality rate of $3 \%$, a 90 -day major morbidity of $13 \%$ and an overall 5 -year survival of $38 \%$ (24).

Careful patient selection, multidisciplinary involvement and team expertise are, in our view, essential in order to reduce peri- and post-operative morbidity and mortality.

We are aware of the limitations of our study, which are mainly related to the retrospective and multicenter nature design. This also includes a certain selection bias. Several outcomes (such as quality of life, return to daily activity) could have been relevant for our analysis but they were not available for all centers due to the nature of the study. Even the indication for neoadjuvant treatment might differ between all the centers due to the heterogeneity of therapeutical protocols. Furthermore, this study is based on databases and data reviews originally collected for clinical and/or quality improvement purposes, and our analysis was limited by the data available. The diversity in practice between centers and countries could have influence outcomes but, at the same time, could provide representative and generalizable data in the thoracic surgery literature on this specific subject.

In conclusion, in patients older than 70 years old, age alone should not be considered as an absolute contraindication for pneumonectomy. It is essential to 
discuss the possible benefits as well as the risks of a surgical approach in a multidisciplinary team approach, along with oncological indications, taking into consideration the comorbidities that often affect this age group of patients.

Only a thorough planning will result in an improvement of outcomes discarding the dogma of "no surgery for the elderly".

\section{Acknowledgments}

The authors would like to thank all members of the Departments of Thoracic Surgery, anesthesiologists, intensive care physicians and pulmonologists involved in the Lung Cancer Programs. At the University Hospitals Leuven we acknowledge the surgical contributions by professor P. De Leyn, professor D. Van Raemdonck and professor H. Decaluwé.

Funding: None.

\section{Footnote}

Reporting Checklist: The authors have completed the STROBE reporting checklist. Available at https://dx.doi. org/10.21037/jtd-21-869

Data Sharing Statement: Available at https://dx.doi. org/10.21037/jtd-21-869

Peer Review File: Available at https://dx.doi.org/10.21037/ jtd-21-869

Conflicts of Interest: All authors have completed the ICMJE uniform disclosure form (available at https://dx.doi. org/10.21037/jtd-21-869). MS serves as an unpaid editorial board member of Fournal of Thoracic Disease from Mar 2020 to Feb 2022. YS serves as an unpaid editorial board member of Fournal of Thoracic Disease. The other authors have no conflicts of interest to declare.

Ethical Statement: The authors are accountable for all aspects of the work in ensuring that questions related to the accuracy or integrity of any part of the work are appropriately investigated and resolved. The study was conducted in accordance with the Declaration of Helsinki (as revised in 2013). The study was approved by the Ethics Board Central Switzerland, project \#2019-02258 and University Hospitals Leuven, Belgium project S63068. Individual consent for this retrospective analysis was waived.
Open Access Statement: This is an Open Access article distributed in accordance with the Creative Commons Attribution-NonCommercial-NoDerivs 4.0 International License (CC BY-NC-ND 4.0), which permits the noncommercial replication and distribution of the article with the strict proviso that no changes or edits are made and the original work is properly cited (including links to both the formal publication through the relevant DOI and the license). See: https://creativecommons.org/licenses/by-nc-nd/4.0/.

\section{References}

1. Vannucci F, Gonzalez-Rivas D. Is VATS lobectomy standard of care for operable non-small cell lung cancer? Lung Cancer 2016;100:114-9.

2. Gridelli C, Perrone F, Monfardini S. Lung cancer in the elderly. Eur J Cancer 1997;33:2313-4.

3. Balducci L. Geriatric oncology: challenges for the new century. Eur J Cancer 2000;36:1741-54.

4. Scarci M, Crisci R, Minervini F. Lung surgery in elderly patients: are we doing enough? J Thorac Dis 2018;10:693-4.

5. Gonfiotti A, Viggiano D, Voltolini L, et al. Enhanced recovery after surgery and video-assisted thoracic surgery lobectomy: the Italian VATS Group surgical protocol. J Thorac Dis 2018;10:S564-70.

6. Jemal A, Siegel R, Ward E, et al. Cancer statistics, 2006. CA Cancer J Clin 2006;56:106-30.

7. Dell'Amore A, Monteverde M, Martucci N, et al. Early and long-term results of pulmonary resection for nonsmall-cell lung cancer in patients over 75 years of age: a multi-institutional study. Interact Cardiovasc Thorac Surg 2013;16:250-6.

8. Bernet F, Brodbeck R, Guenin MO, et al. Age does not influence early and late tumor-related outcome for bronchogenic carcinoma. Ann Thorac Surg 2000;69:913-8.

9. Yamamoto K, Padilla Alarcón J, Calvo Medina V, et al. Surgical results of stage I non-small cell lung cancer: comparison between elderly and younger patients. Eur J Cardiothorac Surg 2003;23:21-5.

10. Sirbu H, Schreiner W, Dalichau H, et al. Surgery for non-small cell carcinoma in geriatric patients: 15 -year experience. Asian Cardiovasc Thorac Ann 2005;13:330-6.

11. Kiser AC, Detterbeck FC. General aspect of surgical treatment. In: Detterbeck FC, Rivera MP, Socinski M, Rosenman JE, editors. Diagnosis and treatment of lung cancer. Philadelphia: WB Saunders, 2001:141-3. 
12. Schneider L, Farrokhyar F, Schieman C, et al. Pneumonectomy: the burden of death after discharge and predictors of surgical mortality. Ann Thorac Surg 2014;98:1976-81; discussion 1981-2.

13. Ljungqvist O, Scott M, Fearon KC. Enhanced Recovery After Surgery: A Review. JAMA Surg 2017;152:292-8.

14. Cerfolio RJ, Pickens A, Bass C, et al. Fast-tracking pulmonary resections. J Thorac Cardiovasc Surg 2001;122:318-24.

15. Muehling BM, Halter GL, Schelzig H, et al. Reduction of postoperative pulmonary complications after lung surgery using a fast track clinical pathway. Eur J Cardiothorac Surg 2008;34:174-80.

16. Gonzalez M, Abdelnour-Berchtold E, Perentes JY, et al. An enhanced recovery after surgery program for videoassisted thoracoscopic surgery anatomical lung resections is cost-effective. J Thorac Dis 2018;10:5879-88.

17. Bilimoria KY, Cohen ME, Ingraham AM, et al. Effect of postdischarge morbidity and mortality on comparisons of hospital surgical quality. Ann Surg 2010;252:183-90.

18. Damhuis RA, Wijnhoven BP, Plaisier PW, et al. Comparison of 30-day, 90-day and in-hospital postoperative mortality for eight different cancer types. $\mathrm{Br}$
J Surg 2012;99:1149-54.

19. Alexiou C, Beggs D, Rogers ML, et al. Pneumonectomy for non-small cell lung cancer: predictors of operative mortality and survival. Eur J Cardiothorac Surg 2001;20:476-80.

20. Sakakura N, Mizuno T, Kuroda H, et al. Primary pneumonectomy, pneumonectomy after induction therapy, and salvage pneumonectomy: a comparison of surgical and prognostic outcomes. J Thorac Dis 2020;12:2672-82.

21. Martin J, Ginsberg RJ, Abolhoda A, et al. Morbidity and mortality after neoadjuvant therapy for lung cancer: the risks of right pneumonectomy. Ann Thorac Surg 2001;72:1149-54.

22. Stamatis G. Risks of neoadjuvant chemotherapy and radiation therapy. Thorac Surg Clin 2008;18:71-80.

23. Albain KS, Swann RS, Rusch VW, et al. Radiotherapy plus chemotherapy with or without surgical resection for stage III non-small-cell lung cancer: a phase III randomised controlled trial. Lancet 2009;374:379-86.

24. Weder W, Collaud S, Eberhardt WE, et al. Pneumonectomy is a valuable treatment option after neoadjuvant therapy for stage III non-small-cell lung cancer. J Thorac Cardiovasc Surg 2010;139:1424-30.
Cite this article as: Minervini F, Kocher GJ, Bertoglio P, Kestenholz PB, Gálvez Muñoz C, Patrini D, Ceulemans LJ, Begum H, Lutz J, Shojai M, Shargall Y, Scarci M. Pneumonectomy for lung cancer in the elderly: lessons learned from a multicenter study. J Thorac Dis 2021;13(10):5835-5842. doi: $10.21037 /$ jtd-21-869 